\title{
A Remark on the Elliptic Boundary Value Problem in an Angular Domain
}

By

\author{
Kazunari HAYASHIDA*
}

1. The general boundary value problems of elliptic equations have been studied quite extensively by several authors (cf., e.g., $[1,2,5,21]$ ). The method is based on the a priori estimates. Most authors have restricted themselves to domains with sufficiently smooth boundary. Their main tool is to map the neighborhood of a point on the boundary onto a semisphere by means of a sufficiently smooth transformation.

On the other hand Ladyzhenskaya [13], [14] and others (cf., e.g., $[9,12]$ ) showed the a priori estimates for domains with piecewise smooth surfaces. Their method is integration by parts. Hence the operators need to be at most of second orders and to be real valued. In this note, assuming a relation between the domain and the elliptic operator, we study $L^{2}$-a priori estimates with a parameter for the second order operators with mixed boundary conditions in an angular domain (see Theorems 1,2 and 3 in the following section).

The a priori estimate with a parameter has been treated in $[2],[4]$, [5] and [10]. In addition the mixed boundary value problem has been studied by several authors (cf. $[17,18,22,23,24]$ ). Our proof relies upon mainly their results.

2. Let $\mathscr{D}$ be a bounded domain in the plane and denote its closure and boundary by $\overline{\mathscr{D}}$ and $\partial \mathscr{D}$. We assume that $\partial \mathscr{D}$ is of class $C^{\infty}$ except a finite number of points $\left\{P_{1}, \ldots, P_{n}\right\}$. Thus $\partial \mathscr{D}=\bigcup_{j=1}^{n} \Gamma_{j}$, where $\Gamma_{j}=\overparen{P}_{j} P_{j+1}$

Received February 6, 1970.

Communicated by S. Matsuura.

* Department of Mathematics, Nagoya University. 
$(j \neq n)$ and $\Gamma_{n}=\overparen{P_{n} P_{1}}$. Let $A\left(x, \frac{\partial}{\partial x}\right)$ be a second order elliptic operator with $C^{\infty}$ coefficients in $\overline{\mathscr{D}}$. And for each $j$ let $B_{j}\left(x, \frac{\partial}{\partial x}\right)$ be a boundary operator of at most first order defined on $\Gamma_{j}$ with $C^{\infty}$ coefficients. We denote the principal parts of $A$ and $B_{j}$ by $A^{(0)}$ and $B_{j}^{(0)}$, respectively. Further let $\mathscr{D}_{j}$ be an angular domain bounded by the two half lines tangent to $\Gamma_{j-1}$ and $\Gamma_{j}$ at $P_{j}$.

First we impose the following condition on $\mathscr{D}$ and $A$.

Condition $\left(A_{1}\right)$. For each $j$ there exists a non singular linear transformation $T_{j}$ such that $A^{(0)}\left(P_{j}, \frac{\partial}{\partial x}\right)$ is transformed into $\Delta\left(=\frac{\partial^{2}}{\partial x_{1}^{2}}+\frac{\partial^{2}}{\partial x_{2}^{2}}\right)$ by $T_{j}$ and $\mathscr{D}_{j}$ is mapped onto an angular domain whose angle is $\frac{\pi}{2}+\alpha$ $\left(|\alpha| \leqq \Theta_{j}\right)$, where $\Theta_{j}$ is a positive constant depending on $A^{(0)}\left(P_{j}, \frac{\partial}{\partial x}\right)$, $B_{j-1}^{(0)}\left(P_{j}, \frac{\partial}{\partial x}\right), B_{j}^{(0)}\left(P_{j}, \frac{\partial}{\partial x}\right)$ and $\mathscr{D}_{j}$.

Secondly we assume the following condition for the boundary operators $B_{j}$.

Condition $\left(A_{2}\right)$. For each $j$ the pair of $B_{j-1}$ and $B_{j}$ corresponds to one of the following cases:

(i) $\quad B_{j-1}=1, \quad \mathrm{~B}_{j}=1$

(ii) $\quad B_{j-1}=1, \quad B_{j}=\frac{\partial}{\partial n}+b_{j}(x)$

(iii) $\quad B_{j-1}=\frac{\partial}{\partial n}+b_{j-1}(x), \quad B_{j}=\frac{\partial}{\partial n}+b_{j}(x)$

(iv) $\quad B_{j-1}=1, \quad B_{j}=\frac{\partial}{\partial n}+a_{j}(x) \frac{\partial}{\partial \tau}+b_{j}(x)$

(v) $\quad B_{j-1}=\frac{\partial}{\partial n}+b_{j-1}(x), \quad B_{j}=\frac{\partial}{\partial n}+a_{j}(x) \frac{\partial}{\partial \tau}+b_{j}(x)$, where $\frac{\partial}{\partial n}$ (or $\frac{\partial}{\partial \tau}$ ) is the normal (or tangent) derivative.

For a fixed real number $\theta$, we set $\lambda^{2}=\rho^{2} e^{i \theta}(0 \leqq \rho<\infty)$. Finally we impose the following assumption for $\theta$ and $a_{j}(x)$ (the coefficients in Condition $\left(A_{2}\right)$ ). 
Condition $\left(A_{3}\right)$.

(i) The boundary operator $B_{j}$ satisfies the Complementing Condition on $\Gamma_{j}$ with respect to $A$ in the sense of $[1]$.

(ii) $e^{i \theta} \neq-1$ and $a_{j}\left(P_{j}\right) \neq \pm i$.

(iii) The value $e^{i \theta}\left(a_{j}\left(P_{j}\right)^{2}+1\right)^{-1}$ does not lie on the real negative axis.

(iv) The value $\left(i+a_{j}\left(P_{j}\right)\right)^{2}\left(i-a_{j}\left(P_{j}\right)\right)^{-2}$ also does not lie on the real negative axis.

Next we give the definitions of some norm. For an integer $k \geqq 0$ we put

$$
\|u\|_{k, \mathscr{D}}=\sum_{|\alpha| \leqq k}\left(\int_{\mathscr{D}}\left|D^{\alpha} u\right|^{2} d x\right)^{1 / 2}
$$

and

$$
\langle u\rangle_{k, \Gamma_{\jmath}}=\sum_{|\alpha| \leqq k}\left(\int_{\Gamma_{j}}\left|D_{\tau}^{\alpha} u\right|^{2} d x\right)^{1 / 2},
$$

where $D_{\tau}^{\alpha}$ is some tangential operator on $\Gamma_{j}$ of order $|\alpha|$. We put

$$
\langle u\rangle_{k, \partial \mathscr{D}}=\sum_{j=1}^{n}\langle u\rangle_{k, \Gamma_{j}} .
$$

We can define naturally the norms \|\|$_{k}$ and \langle\rangle$_{k}$ also for a real $k \geqq 0$ (cf., e.g., $[18,20,21]$ ). Then we have

Theorem 1. Under the conditions $\left(A_{1}\right),\left(A_{2}\right)$ and $\left(A_{3}\right)$, there are nonnegative constants $k_{0}, c$ and $C$ such that for any $u \in C^{\infty}(\overline{\mathscr{D}})$

$$
\begin{aligned}
\|u\|_{2+k}^{2}+|\lambda|^{2(2+k)}\|u\|_{0}^{2} \leqq & C\left[\left\|\left(A-\lambda^{2}\right) u\right\|_{k}^{2}\right. \\
& +\left.|\lambda|^{2 k}\right|_{i}\left(A-\lambda^{2}\right) u \|_{0}^{2}+\sum_{j}\left(\left\langle B_{j} u\right\rangle_{\frac{3}{2}-m_{j}+k, \Gamma_{j}}^{2}\right. \\
& \left.\left.+|\lambda|^{2\left(\frac{3}{2}-m_{j}+k\right)}\left\langle B_{j} u\right\rangle_{0, \Gamma_{j}}^{2}\right)\right],
\end{aligned}
$$

if $k$ is an integer $\geqq k_{0}$ and $|\lambda| \geqq c$, where $k_{0}$ and $c$ depend on $A, B_{j}$, $\mathscr{D}$ and $\theta$. Further $C$ depends on $A, B_{j}, \mathscr{D}, k$ and $c$.

Theorem 2. Assume that for each $j$ one of the cases (i), (ii) and (iii) in Condition $\left(A_{2}\right)$ holds. Then under the conditions $\left(A_{1}\right)$ and $\left(A_{3}\right)$, 
we can take $k_{0}=0$ in the statement of Theorem 1 .

Remark 1. When for each $j,\left(B_{j-1}, B_{j}\right)$ corresponds to one of the cases (i), (ii) and (iii) in Condition $\left(A_{2}\right)$, it will be seen that we can calculate $\Theta_{j}$ in Condition $\left(A_{1}\right)$ exactly.

Remark 2. Let $B_{j}$ be smooth on $\Gamma_{j}$ except at most finite points, that is,

$$
B_{j}=b_{j}(x) \frac{\partial}{\partial n}+a_{j}(x) \frac{\partial}{\partial \tau}+c_{j}(x)
$$

where $a_{j}, b_{j}, c_{j}$ are piecewise smooth and some conditions are assumed. Then Theorem 1 holds also according to Shamir's [18], Agmon's [2] and our arguments.

Theorem 3. Under the above conditions assume that $k_{0}$ can be taken to be 0 . Then for any $f(x) \in C^{\infty}(\overline{\mathscr{D}})$, there are solutions $u \in C^{\infty}\left(\overline{\mathscr{D}}-\bigcup_{i=1}^{n} P_{i}\right) \cap L^{2}(\mathscr{D})$ satisfying

$$
\begin{gathered}
\left(A^{*}-\bar{\lambda}^{2}\right) u=f \quad \text { in } \mathscr{D} \quad(|\lambda|>c) \\
B_{j}^{\prime} u=0 \quad \text { on } \quad \Gamma_{j}-\left(P_{j-1} \cup P_{j}\right) \quad \text { for each } j,
\end{gathered}
$$

where $A^{*}$ is the formal adjoint of $A$ and $B_{j}^{\prime}$ is the adjoint operator with respect to $A$ in the sense of Schechter [21].

The proof of Theorem 3 follows immediately from Theorem 2. We make the form

$$
\begin{aligned}
{[u, v]=} & \left(\left(A-\lambda^{2}\right) u,\left(A-\lambda^{2}\right) v\right)+\sum_{j}\left(\left\langle B_{j} u, B_{j} v\right\rangle_{\frac{3}{2}-m_{j}, \Gamma_{j}}\right. \\
& \left.+|\lambda|^{3-2 m_{j}}\left\langle B_{j} u, B_{j} v\right\rangle_{0, \Gamma_{j}}\right)
\end{aligned}
$$

Then by Theorem 2 it is seen that $[u, u]^{1 / 2}$ defines a norm equivalent to the usual one of $H^{2}(\mathscr{D}) .{ }^{1)}$ Thus we can apply the theorem of Riesz to the functional $(f, v)$ on the space with the norm $[u, u]^{1 / 2}$. The process is quite analogous to the works of Schechter (see [21], [22]).

1) The space $H^{2}(\mathscr{D})$ is the Sobolev space with the norm $\|u\|_{2, \mathscr{D}}$. 
3. We take a real number $\theta$ and a complex number $a$ satisfying the following

Condition $\left(A_{3}^{\prime}\right)$.

(i) $e^{i \theta} \neq-1$ and $a \neq \pm i$.

(ii) $e^{i \theta}\left(a^{2}+1\right)^{-1}$ is not a real negative number.

(iii) $(i+a)^{2}(i-a)^{-2}$ is not a real negative number.

Now we consider the characteristic equation with respect to $\zeta$

$$
\zeta^{2}+\xi^{2}+e^{i \theta} \eta^{2}=0
$$

where $\xi$ and $\eta(\neq 0)$ are real numbers. The root of (3.1) with positive imaginary part is denoted by $\zeta^{+}(\xi, \eta)$.

Set

$$
M(\xi, \eta)=\frac{\zeta^{+}(\xi, \eta)-a \xi}{\zeta^{+}(\xi, \eta)+a \xi}
$$

Then it is seen from the property (ii) of Condition $\left(A_{3}^{\prime}\right)$ that $M(\xi, \eta)$ is homogeneous of degree 0 and does not vanish. We define the Fourier transform

$$
(\mathscr{F} u)(\xi, \eta)=\iint e^{-i\left(\eta t+\xi x_{1}\right)} u\left(x_{1}, t\right) d x_{1} d t
$$

And consider the operator

$$
(\boldsymbol{M} u)\left(x_{1}, t\right)=\left(\mathscr{F}^{-1}(M \cdot \mathscr{F} u)\right)\left(x_{1}, t\right) .
$$

We define the norm \|\|$_{k}$ for $u\left(x_{1}, t\right)$ in the same way as in section 2 . And denote by \|\|$_{k,\left\{x_{1} \gtrless 0\right\}}$ the quotient norm of \|\|$_{k}$ on $x_{1} \gtrless 0$, respectively.

The following proposition is due to Shamir [19].

Proposition 3.1 ([19]). Under the condition $\left(A_{3}^{\prime}\right)$, there are an integer $k_{0}$ and a constant $C$ such that

$$
\|u\|_{k+\frac{1}{2}} \leqq C\left(\|u\|_{k+\frac{1}{2},\left\{x_{1}<0\right\}}+\|\boldsymbol{M} u\|_{k+\frac{1}{2},\left\{x_{1}>0\right\}}\right)
$$


for any $u \in H_{k+\frac{1}{2}}\left(R^{2}\right)$, if $k$ is an integer $\geqq k_{0}$.

In particular Shamir [19] proved the proposition in $L^{p}$ space. His method is due to Gohberg-Krein factorization ( $[7],[8]$ ) such as

$$
M(\xi, \eta)=C Q_{-}^{-1}\left(\xi|\eta|^{-1}\right)(\xi-i|\eta|)^{-\zeta-\kappa}(\xi+i|\eta|)^{\zeta+\kappa} Q_{+}\left(\xi|\eta|^{-1}\right)
$$

where $Q_{-}^{-1}\left(\xi|\eta|^{-1}\right)$ and $Q_{+}\left(\xi|\eta|^{-1}\right)$ are holomorphic in $\operatorname{Im} \xi>0$ and $\operatorname{Im} \xi<0$ respectively. And Gohberg-Krein factorization $([7],[8])$ is produced from Wiener-Lévy's theorem $([25])$ in Riemann Hilbert problem.

4. Let $R^{3}$ be the 3 -dimensional Euclidean space with coordinate $\left(x_{1}, x_{2}, t\right)$. We set

$$
C_{0}^{\infty}\left(\bar{R}_{+}^{3}\right)=\left\{u \in C^{\infty}\left(\bar{R}_{+}^{3}\right) \mid \text { carrier of } u \subset \bar{R}_{+}^{3}\right\},
$$

where $\bar{R}_{+}^{3}=\left\{\left(x_{1}, x_{2}, t\right) \mid x_{2} \geqq 0\right\}$. For an integer $k \geqq 0$ and for any function $u$ defined in $\bar{R}_{+}^{3}$, we define the semi-norm and the norm

$$
\begin{gathered}
|u|_{k}^{2}=\sum_{|\alpha|=k} \int_{0}^{\infty}\left(\iint_{R^{2}}\left|D_{x_{1}}^{\alpha_{1}} D_{x_{2}}^{\alpha_{2}} D_{t}^{\alpha_{3}} u\right|^{2} d x_{1} d t\right) d x_{2}{ }^{2)} \\
\|u\|_{k}^{2}=\sum_{s \leqq k}|u|_{s}^{2} .
\end{gathered}
$$

Let $\hat{u}\left(\xi, x_{2}, \eta\right)$ be the Fourier transform of $u$ in $\left(x_{1}, t\right)$. Then for the trace of $u$, we define the boundary norm

$$
\langle u\rangle_{k+\frac{1}{2}}^{2}=\iint_{R^{2}}\left(\xi^{2}+\eta^{2}\right)^{\left(k+\frac{1}{2}\right)}|\hat{u}(\xi,+0, \eta)|^{2} d \xi d \eta .
$$

Now let us consider the elliptic operator in $R_{+}^{3}$ and the boundary operator on $x_{2}=0$ :

$$
\begin{aligned}
& L=\frac{\partial^{2}}{\partial x_{1}^{2}}+\frac{\partial^{2}}{\partial x_{2}^{2}}+e^{i \theta} \frac{\partial^{2}}{\partial t^{2}} \\
& B_{ \pm}=\frac{\partial}{\partial x_{2}} \pm a \frac{\partial}{\partial x_{1}}
\end{aligned}
$$

where $\theta$ and $a$ satisfy the properties (i) and (ii) in Condition $\left(A_{3}^{\prime}\right)$. We denote the characteristic polynomials of (4.1) by

2) $\alpha=\left(\alpha_{1}, \alpha_{2}, \alpha_{3}\right)$ and $|\alpha|=\alpha_{1}+\alpha_{2}+\alpha_{3}$. 


$$
\begin{aligned}
& L(\xi, \zeta, \eta)=-\left(\zeta^{2}+\xi^{2}+e^{i \theta} \eta^{2}\right) \\
& B_{-}(\xi, \zeta)=i(\zeta-a \xi) .
\end{aligned}
$$

Since $e^{i \theta} \neq-1$, it is easily seen that if $(\xi, \zeta, \eta, \mu) \in R^{4}$, then

$$
\left|L(\xi, \zeta, \eta)-\mu^{2} e^{i \theta}\right| \geqq c\left(\xi^{2}+\zeta^{2}+\eta^{2}+\mu^{2}\right),
$$

where $c$ is a positive constant. For every $(\xi, \eta, \mu)(\neq(0,0,0)) \in R^{3}$ the polynomial in $\zeta L(\xi, \zeta, \eta)-\mu^{2} e^{i \theta}$ has a root $\zeta^{+}(\xi, \eta, \mu)$ with positive imaginary part. Then we easily see that there is a positive constant $c$ such that

$$
\left|\frac{1}{2 \pi i} \oint \frac{B(\xi, \zeta) d \zeta}{\zeta-\zeta^{+}(\xi, \eta, \mu)}\right| \geqq c\left(\xi^{2}+\eta^{2}+\mu^{2}\right)^{1 / 2}
$$

where the integration is taken along a closed curve in the complex $\zeta$-plane enclosing $\zeta^{+}(\xi, \eta, \mu)$.

Under the properties (4.2) and (4.3), Agranovich [4] and Higuchi [10] proved the following proposition.

Proposition 4.1 ([4], $[10])$. Let $\theta$ and a satisfy the assumptions (i), (ii) in Condition $\left(A_{3}^{\prime}\right)$. Then there is a constant $C$ depending only on $\theta$ and $a$ such that for any $u \in C_{0}^{\infty}\left(\bar{R}_{+}^{3}\right)$ and any $\mu>0$,

$$
\begin{aligned}
|u|_{k+2}^{2}+\mu^{4+2 k}|u|_{0}^{2} \leqq & C\left(\left|\left(L-\mu^{2} e^{i \theta} u\right)\right|_{k}^{2}\right. \\
& +\mu^{2 k}\left|\left(L-\mu^{2} e^{i \theta}\right) u\right|_{0}^{2}+\left\langle B_{-} u\right\rangle_{k+\frac{1}{2}}^{2} \\
& \left.+\mu^{1+2 k}\left\langle B_{-} u\right\rangle_{0}^{2}\right) .
\end{aligned}
$$

The $L^{2}$ a priori estimates for mixed problems in $R^{n}$ were obtained by Vishik-Eskin ([23], [24]). But their proof is difficult to follow. Thus we shall give another brief proof for our case.

Proposition 4.2. Under the Condition $\left(A_{3}^{\prime}\right)$, there exist an integer $k_{0}$ and a constant $C$ such that if $k$ is an integer $\geqq k_{0}$,

$$
|u|_{k+2} \leqq C\left(|L u|_{k}+\left\langle B_{-} u\right\rangle_{k+\frac{1}{2},\left\{x_{1}<0\right\}}+\left\langle B_{+} u\right\rangle_{k+\frac{1}{2},\left\{x_{1}>0\right\}}+|u|_{0}\right)
$$

for any $u \in C_{0}^{\infty}\left(\bar{R}_{+}^{3}\right)$, where \langle\rangle$_{k+\frac{1}{2}},\left\{x_{1} \gtrless 0\right\}$ means the quotient boundary norm in $x_{1} \gtrless 0$ respectively. 
Proof. Let us put

$$
M(\xi, \eta, \mu)=\frac{\zeta^{+}(\xi, \eta, \mu)+a \xi}{\zeta^{+}(\xi, \eta, \mu)-a \xi} .
$$

Since the property (ii) of Condition $\left(A_{3}^{\prime}\right)$ holds, it is easily seen that $\zeta^{+}(\xi, \eta, \mu)-a \xi \neq 0$ if $(\xi, \eta, \mu) \neq(0,0,0)$ and $\in R^{3}$. Thus $M(\xi, \eta, \mu)$ is uniformly bounded for nonzero real vectors $(\xi, \eta, \mu)$. We define the Fourier transform

$$
(\mathscr{F} u)(\xi, \eta)=\iint e^{i\left(\xi x_{1}+\eta t\right)} u\left(x_{1}, t\right) d x_{1} d t
$$

Put

$$
\boldsymbol{M}=\mathscr{F}^{-1} M(\xi, \eta, 0) \mathscr{F}, \quad \boldsymbol{M}_{\mu}=\mathscr{F}^{-1} M(\xi, \eta, \mu) \mathscr{F} .
$$

Then we shall prove the following inequality:

$$
\begin{aligned}
\left\langle B_{-} u\right\rangle_{k+\frac{1}{2}}^{2} \leqq & C\left(\left\langle B_{-} u\right\rangle_{k+\frac{1}{2}}^{2},\left\{x_{1}<0\right\}\right. \\
& +\left|\left(L-\mu^{2} e^{i \theta}\right) u\right|_{k}^{2}+\left\langle\left(\boldsymbol{M}-\boldsymbol{B}_{+} u\right\rangle_{k+\frac{1}{2}}^{2},\left\{x_{1}\right\rangle 0\right\} \\
& \left.\left.+B_{-} u\right\rangle_{k^{+}}^{2}+|u|_{0}^{2}\right)
\end{aligned}
$$

Now it is well known that there is an extension $F\left(x_{1}, x_{2}, t ; \mu\right) \in C_{0}^{s}\left(R^{3}\right)$ (for sufficiently large $s$ ) of $\left(L-\mu^{2} e^{i \theta}\right) u \in C_{0}^{\infty}\left(\bar{R}_{+}^{3}\right)$ such that

$$
|F|_{m} \leqq C\left|\left(L-\mu^{2} e^{i \theta}\right) u\right|_{m} \quad(m \leqq s),
$$

where $C$ is a constant depending only on $m$. We set

$$
\begin{aligned}
& f\left(x_{1}, t\right)=\left(B_{-} u\right)\left(x_{1}, 0, t\right), \\
& \hat{u}(\xi, \eta)=(\mathscr{F} u)(\xi, \eta) \quad(\text { see }(4.5))
\end{aligned}
$$

and

$$
\tilde{F}(\xi, \zeta, \eta ; \mu)=\int e^{-i\left(\xi x_{1}+\zeta x_{2}+\eta t\right)} F\left(x_{1}, x_{2}, t ; \mu\right) d x_{1} d x_{2} d t
$$

Then any $u \in C_{0}^{\infty}\left(R_{+}^{3}\right)$ can be written in the form

$$
u\left(x_{1}, x_{2}, t\right)=w\left(x_{1}, x_{2}, t ; \mu\right)+v\left(x_{1}, x_{2}, t ; \mu\right),
$$

where 


$$
\hat{w}\left(\xi, \eta, x_{2} ; \mu\right)=\int_{-\infty}^{\infty} \frac{e^{i x_{2 \zeta} \zeta}}{\zeta^{2}+\xi^{2}+e^{i \theta} \eta^{2}+\mu^{2}} \tilde{F}(\xi, \zeta, \eta ; \mu) d \zeta
$$

and

$$
\begin{aligned}
\hat{v}\left(\xi, \eta, x_{2} ; \mu\right)= & \left(\hat{f}(\xi, \eta)-B_{-}\left(i \xi, \frac{\partial}{\partial x_{2}}\right) \hat{w}(\xi, \eta, 0 ; \mu)\right) \cdot \\
& \frac{e^{i x_{2} \zeta^{+}(\xi, \eta, \mu)}}{i B_{-}\left(\xi, \zeta^{+}(\xi, \eta, \mu)\right)} .
\end{aligned}
$$

The decomposition (4.7) was used to prove Proposition 4.1 by Agranovich [4] and Higuchi [10].

From (4.9) we see

$$
\begin{aligned}
& B_{+}\left(i \xi, \frac{\partial}{\partial x_{2}}\right) \hat{v}(\xi, \eta, 0 ; \mu) \\
& \quad=\left(\hat{f}(\xi, \eta)-B_{-}\left(i \xi, \frac{\partial}{\partial x_{2}}\right) \hat{w}(\xi, \eta, 0 ; \mu)\right) M(\xi, \eta, \mu) .
\end{aligned}
$$

By Proposition 3.1,

$$
\langle f\rangle_{k+\frac{1}{2}}^{2} \leqq C\left(\langle f\rangle_{k+\frac{1}{2}}^{2},\left\{x_{1}<0\right\}+\langle\boldsymbol{M} f\rangle_{k+\frac{1}{2}}^{2},\left\{x_{1}>0\right\}\langle f\rangle_{0}^{2}\right),
$$

if $k \geqq k_{0}$. This inequality implies

$$
\begin{aligned}
\langle f\rangle_{k+}^{2} \leqq & C\left(\langle f\rangle_{k+\frac{1}{2}}^{2},\left\{x_{1}<0\right\}\right. \\
& +\left\langle\boldsymbol{M}_{\mu} f\right\rangle_{k+\frac{1}{2}}^{2},\left\{x_{1}>0\right\} \\
& \left.+\left\langle\left(\boldsymbol{M}-\boldsymbol{M}_{\mu}\right) f\right\rangle_{k+\frac{1}{2}}^{2},\left\{x_{1}>0\right\}\langle f\rangle_{0}^{2}\right) .
\end{aligned}
$$

Since $M(\xi, \eta, \mu)$ is uniformly bounded, we see from (4.10)

$$
\begin{aligned}
\left\langle\boldsymbol{M}_{\mu} f\right\rangle_{k+\frac{1}{2}}^{2},\left\{x_{1}>0\right\} \leqq & C\left(\left\langle B_{+} v\right\rangle_{k+\frac{1}{2}}^{2},\left\{x_{1}\right\rangle 0\right\} \\
& \left.+\left\langle B_{-} w\right\rangle_{k+\frac{1}{2}}^{2}\right) .
\end{aligned}
$$

In view of (4.7) and (4.8),

$$
\left\langle\boldsymbol{M}_{\mu} f\right\rangle_{k+\frac{1}{2}}^{2},\left\{x_{1}>0\right\} \leqq C\left(\left\langle B_{+} u\right\rangle_{k+\frac{1}{2}}^{2},\left\{x_{1}>0\right\}\right.
$$

$$
\left.+\left|\left(L-\mu^{2} e^{i \theta}\right) u\right|_{k}^{2}+\mu^{2 k}\left|\left(L-\mu^{2} e^{i \theta}\right) u\right|_{0}^{2}\right) .
$$

Combining (4.11) and (4.12), we have proved (4.6). 
By Proposition 4.1 and (4.6), we obtain

$$
\begin{aligned}
|u|_{k+2}^{2}+\mu^{4+2 k}|u|_{0}^{2} \leqq & C\left(\left|\left(L-\mu^{2} e^{i \theta}\right) u\right|_{k}^{2}\right. \\
& +\mu^{2 k}\left|\left(L-\mu^{2} e^{i \theta}\right) u\right|_{0}^{2}+\left\langle B_{-} u\right\rangle_{k+\frac{1}{2}}^{2},\left\{x_{1}<0\right\} \\
& +\left\langle B_{+} u\right\rangle_{k+\frac{1}{2}}^{2},\left\{x_{1}>0\right\}+\mu^{1+2 k}\left\langle B_{-} u\right\rangle_{0}^{2} \\
& \left.+\left\langle\left(\boldsymbol{M}-\boldsymbol{M}_{\mu}\right) f\right\rangle_{k+\frac{1}{2}}^{2}\right) .
\end{aligned}
$$

Obviously,

$$
\begin{aligned}
\left\langle\left(\boldsymbol{M}-\boldsymbol{M}_{\mu}\right) f\right\rangle_{k+\frac{1}{2}}^{2} \leqq & C \iint_{\substack{\xi^{2}+\eta^{2} \leq \delta \\
\xi^{2}+\eta^{2} \geqq K}}\left(\xi^{2}+\eta^{2}\right)^{k+\frac{1}{2}}|\hat{f}(\xi, \eta)|^{2} d \xi d \eta \\
& +C \iint_{\delta \leqq \xi^{2}+\eta^{2} \leqq K}\left(\xi^{2}+\eta^{2}\right)^{k+\frac{1}{2}} \mid M(\xi, \eta, 0) \\
& -\left.M(\xi, \eta, \mu)\right|^{2}|\hat{f}(\xi, \eta)|^{2} d \xi d \eta
\end{aligned}
$$

Taking $\delta$ as sufficiently small and $K$ as sufficiently large, we note that $M(\xi, \eta, \mu)$ tends to $M(\xi, \eta, 0)$ uniformly as $\mu \rightarrow 0$ in $\delta \leqq \xi^{2}+\eta^{2} \leqq K$. Then we see from (4.14)

$$
\left\langle\left(\boldsymbol{M}-\mathbf{M}_{\mu}\right) f\right\rangle_{k+\frac{1}{2}} \rightarrow 0 \quad \text { as } \quad \mu \rightarrow 0 .
$$

Thus taking $\mu \rightarrow 0$ in (4.13), we have completed the proof.

5. In this section we also consider the operators $L$ and $B_{ \pm}$as in (4.1). And let $\theta$ and $a$ satisfy the condition $\left(A_{3}^{\prime}\right)$. The following proposition is due to $[4]$ and $[10]$ essentially as in Proposition 4.1.

Proposition 5.1. For any given $g_{j}\left(x_{1}, t\right) \in C_{0}^{\infty}\left(R^{2}\right)(1 \leqq j \leqq l)$, there is a solution $u \in \mathscr{S}\left(\bar{R}_{+}^{3}\right)^{3)}$ such that

$$
\begin{aligned}
& \left(L-\mu^{2} e^{i \theta}\right)^{l} u=0 \quad \text { in } \quad R_{+}^{3} \quad(\mu>0) \\
& \frac{\partial^{j-1}}{\partial x_{2}^{j-1}} u\left(x_{1}, 0, t\right)=g_{j} \quad \text { on } \quad x_{2}=0 . \\
& |u|_{2 l+s}^{2}+\mu^{2(2 l+s)}|u|_{0}^{2} \leqq C \sum_{j=1}^{l}\left(\left\langle g_{j}\right\rangle_{2 l+s-j+\frac{1}{2}}^{2}\right.
\end{aligned}
$$

3) The letter $\mathscr{Y}\left(\bar{R}_{+}^{3}\right)$ means the set of $C^{\infty}$ functions decreasing rapidly in $\bar{R}_{+}^{3}$. 


$$
\left.+\mu^{2\left(2 l+s-j+\frac{1}{2}\right)}\left\langle g_{j}\right\rangle_{0}^{2}\right)
$$

where $l+s \geqq 0$.

Remark. If $s \geqq 0$, the estimate (5.1) holds for general boundary operators. In particular under the Dirichlet boundary condition, the assumption on $s$ is weakened as in Proposition 5.1. For more general negative $s$, the a priori estimate without parameters was shown by Lions and Magenes ([15], [16]). Their method is due to the closed graph theorem and Gårding's inequality ([3], $[6])$.

Lemma 5.1. Let $u$ be in $C_{0}^{m-1}\left(\bar{R}_{3}^{+}\right)$and be in $C_{0}^{m}\left(\bar{R}_{3}^{+}\right)$except on $x_{1}=0$. Then if $D^{m} u^{4)}$ is bounded, there is a sequence $\left\{u_{n}\right\}$ such that $u_{n}$ belongs to $C_{0}^{m}\left(\bar{R}_{3}^{+}\right)$and $\left\|u_{n}-u\right\|_{m} \rightarrow 0$ as $n \rightarrow \infty$.

Proof. Let $J_{\varepsilon}$ be the mollifier and let us set

$$
u_{\varepsilon}\left(x_{1}, x_{2}, t\right)=\int J_{\varepsilon}\left(x_{1}-x_{1}^{\prime}\right) u\left(x_{1}^{\prime}, x_{2}, t\right) d x_{1}^{\prime} .
$$

By integration by parts, we see

$$
\begin{aligned}
\left(D^{m} u_{\varepsilon}\right)\left(x_{1}, x_{2}, t\right) & =\int J_{\varepsilon}\left(x_{1}-x_{1}^{\prime}\right) D^{m} u\left(x_{1}^{\prime}, x_{2}, t\right) d x_{1}^{\prime} \\
& =\int J_{\varepsilon}\left(x_{1}^{\prime}\right) D^{m} u\left(x_{1}-x_{1}^{\prime}, x_{2}, t\right) d x_{1}^{\prime} .
\end{aligned}
$$

Obviously,

$$
\left(D^{m} u\right)\left(x_{1}, x_{2}, t\right)=\int J_{\varepsilon}\left(x_{1}^{\prime}\right) D^{m} u\left(x_{1}, x_{2}, t\right) d x_{1}^{\prime} .
$$

Hence,

$$
\left\|D^{m} u_{\varepsilon}-D^{m} u\right\|_{0} \rightarrow 0 \quad \text { as } \quad \varepsilon \rightarrow 0 .
$$

Thus we have proved the lemma.

Let us put $\Sigma_{ \pm}=\left\{\left(x_{1}, x_{2}, t\right) \mid x_{1} \gtrless 0, x_{2}>0\right\}$ and $\Gamma=\left\{\left(0, x_{2}, t\right) \mid x_{2} \geq 0\right\}$. We introduce the function space:

$C_{0}^{m}\left(\bar{\Sigma}_{+}\right)=\left\{u \in C^{m}\left(\bar{\Sigma}_{+}\right) \mid u\right.$ vanishes for sufficiently large $\left.x_{1}^{2}+x_{2}^{2}+t^{2}\right\}$.

4) We denote by $D^{m} u$ some derivative of $u$ of order $m$. 
The following a priori estimate hold for $C_{0}^{m}\left(\bar{\Sigma}_{+}\right)$:

Proposition 5.2. Under the above assumptions, if $k \geqq k_{0}\left(k_{0}\right.$ is the same constant as in Proposition 4.2), it holds that for any $u \in C_{0}^{\infty}\left(\bar{\Sigma}_{+}\right)$

$$
\begin{aligned}
|u|_{k+2, \Sigma_{+}} \leqq & C\left(|L u|_{k, \Sigma_{+}}+\left\langle u_{x_{1}}\right\rangle_{k+\frac{1}{2}, \Gamma}\right. \\
& \left.+\left\langle u_{x_{2}}+a u_{x_{1}}\right\rangle_{k+\frac{1}{2},\left\{x_{1}>0\right\}}+|u|_{0}\right) .^{5)}
\end{aligned}
$$

Proof. We extend the functions $D_{x_{1}}^{m} u\left(0, x_{2}, t\right)$ to the whole plane $x_{1}=0$ for $m \leqq k+1$ in such a way that

$$
\tilde{u}_{m}\left(x_{2}, t\right)=D_{x_{1}}^{m} u\left(0, x_{2}, t\right) \quad \text { in } \quad x_{2} \geqq 0, \quad \tilde{u}_{m} \in C_{0}^{k+2}\left(R^{2}\right)
$$

and

$$
\begin{aligned}
& \left\langle\tilde{u}_{m}\right\rangle_{k+\frac{3}{2}-m,\left\{x_{1}=0\right\}} \leqq C\left\langle D_{x_{1}}^{m} u\right\rangle_{k+\frac{3}{2}-m, \Gamma} \\
& \left\langle\tilde{u}_{m}\right\rangle_{0,\left\{x_{1}=0\right\}} \leqq C\left\langle D_{x_{1}}^{m} u\right\rangle_{0, \Gamma} .
\end{aligned}
$$

Hereafter we shall denote $\tilde{u}_{m}$ simply by $D_{x_{1}}^{m} u$.

Now by Proposition 5.1 there is a solution $w \in \mathscr{S}\left(x_{1} \leqq 0\right)^{6)}$ such that

$$
\left(L-\mu^{2} e^{i \theta}\right)^{2+k} w=0 \quad \text { in } \quad x_{1}<0
$$

and for $0 \leqq m \leqq k+1$

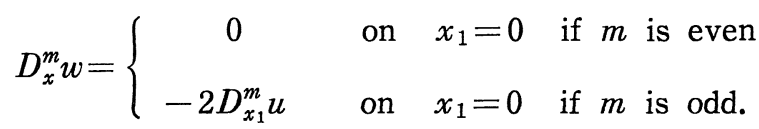

Put

$$
v\left(x_{1}, x_{2}, t\right)=u\left(-x_{1}, x_{2}, t\right)-w\left(x_{1}, x_{2}, t\right) \quad \text { for } \quad x_{1} \leqq 0 .
$$

And we define

$$
\tilde{u}\left(x_{1}, x_{2}, t\right)= \begin{cases}u\left(x_{1}, x_{2}, t\right) & \text { for } \quad x_{1} \geqq 0 \\ v\left(x_{1}, x_{2}, t\right) & \text { for } \quad x_{1}<0 .\end{cases}
$$

Then by (5.4) the function $u$ satisfies the assumption of Lemma 5.1 for

5) The notation \langle\rangle$_{s,\left\{x_{1}>0\right\}}$ is the quotient $s$ semi-norm in the half plane $\left\{\left(x_{1}, 0, t\right) \mid x_{1}>0\right\}$.

6) We denote by $y\left(x_{1} \leqq 0\right)$ the set of $C^{\infty}$ functions decreasing rapidly in $x_{1} \leqq 0$. 
$m=k+2$. Hence taking the approximating sequence, we have by Proposition 4.2

$$
\begin{aligned}
|\tilde{u}|_{k+2} \leqq & C\left(|L \tilde{u}|_{k}+\left\langle\tilde{u}_{x_{2}}-a \tilde{u}_{x_{1}}\right\rangle_{k+\frac{1}{2},\left\{x_{1}<0\right\}}\right. \\
& \left.+\left\langle\tilde{u}_{x_{2}}+a \tilde{u}_{x_{1}}\right\rangle_{k+\frac{1}{2},\left\{x_{1}>0\right\}}+|u|_{0}\right) .
\end{aligned}
$$

Obviously,

$$
|L \tilde{u}|_{k} \leqq|L u|_{k, \Sigma_{+}}+|L v|_{k, \Sigma_{-}} \leqq 2|L u|_{k, \Sigma_{+}}+|L w|_{k, \Sigma_{-}}
$$

and

$$
\begin{aligned}
\left\langle\tilde{u}_{x_{2}}-a \tilde{u}_{x_{1}}\right\rangle_{k+\frac{1}{2}},\left\{x_{1}<0\right\} \leqq & \left\langle u_{x_{2}}+a u_{x_{1}}\right\rangle_{k+\frac{1}{2}},\left\{x_{1}>0\right\} \\
& +\left\langle w_{x_{2}}-a w_{x_{1}}\right\rangle_{k+\frac{1}{2}},\left\{x_{1}<0\right\}
\end{aligned}
$$

In view of Proposition 5.1 and (5.4), we get

$$
\begin{aligned}
|w|_{2+k,\left\{x_{1}<0\right\}}^{2} \leqq & C \sum_{j=0}^{\left[\frac{k}{2}\right]^{7)}}\left(\left\langle D_{x_{1}}^{2 j+1} u\right\rangle_{k-2 j+\frac{1}{2}, \Gamma}^{2}\right. \\
& \left.+\mu^{2\left(k-2 j+\frac{1}{2}\right)}\left\langle D_{x_{1}}^{2 j+1} u\right\rangle_{0, \Gamma}^{2}\right),
\end{aligned}
$$

where we have put $s=-l$ in (5.1).

Since $D_{x_{1}}^{2}=L-\left(D_{x_{2}}^{2}+e^{i \theta} D_{t}^{2}\right)$, we see

$$
D_{x_{1}}^{2 j}=\sum_{j_{1}+j_{2}=j} c_{j_{1}, j_{2}} L^{j_{1}}\left(D_{x_{2}}^{2}+e^{i \theta} D_{t}^{2}\right)^{j_{2}}
$$

Therefore,

$$
\left\langle D_{x_{1}}^{2 j+1} u\right\rangle_{k-2 j+\frac{1}{2}, r} \leqq C \sum_{j_{1}+j_{2}=j}\left\langle D_{x_{1}} L^{j_{1}} u\right\rangle_{k-2 j+\frac{1}{2}+2 j_{2}, \Gamma} .
$$

If $j_{1}=0$ on the term on the right side, we easily see

$$
\left\langle D_{x_{1}} L^{j_{1}} u\right\rangle_{k-2 j+\frac{1}{2}+2 j_{2}, \Gamma} \leqq\left\langle D_{x_{1}} u\right\rangle_{k+\frac{1}{2}, \Gamma}
$$

If $j_{1} \neq 0$, we have by the well known inequality (see e.g., [11])

$$
\begin{aligned}
\left\langle D_{x_{1}} L^{j_{1}} u\right\rangle_{k-2 j+\frac{1}{2}+2 j_{2}, \Gamma} & \leqq C\left|D_{x_{1}} L^{j_{1}} u\right|_{k-2 j+1+2 j_{2}, \Sigma_{+}} \\
& \leqq C|L u|_{k, \Sigma_{+}} .
\end{aligned}
$$

7) The notation $[s]$ means the integral part of $s$. 
Hence we get from (5.10) and (5.11)

$$
\left\langle D_{x_{1}} L^{j_{1}} u\right\rangle_{k-2 j+\frac{1}{2}+2 j_{2}, \Gamma} \leqq C\left(|L u|_{k, \Sigma_{+}}+\left\langle D_{x_{1}} u\right\rangle_{k+\frac{1}{2}, \Gamma}\right)
$$

We see from (5.8), (5.9) and (5.12)

$$
\begin{aligned}
|w|_{k+2,\left\{x_{1}<0\right\}}^{2} \leqq & C\left(|L u|_{k, \Sigma_{+}}^{2}+\left\langle D_{x_{1}} u\right\rangle_{k+\frac{1}{2}, \Gamma}^{2}\right. \\
& \left.+\sum_{j=0}^{\left[\frac{k}{2}\right]} \mu^{2\left(k-2 j+\frac{1}{2}\right)}\left\langle D_{x_{1}}^{2 j+1} u\right\rangle_{0, \Gamma}^{2}\right) .
\end{aligned}
$$

On the other hand we get by (5.5), (5.6) and (5.7)

$$
\begin{aligned}
|u|_{k+2, \Sigma_{+}} \leqq & C\left(|L u|_{k, \Sigma_{+}}+\left\langle u_{x_{2}}+a u_{x_{1}}\right\rangle_{k+\frac{1}{2}},\left\{x_{1}>0\right\}\right. \\
& \left.+|L w|_{k,\left\{x_{1}<0\right\}}+\left\langle w_{x_{2}}-a w_{x_{1}}\right\rangle_{k+\frac{1}{2},\left\{x_{1}<0\right\}}+|u|_{0}\right) .
\end{aligned}
$$

Obviously,

$$
|L w|_{k,\left\{x_{1}<0\right\}},\left\langle w_{x_{2}}-a w_{x_{1}}\right\rangle_{k+\frac{1}{2}},\left\{x_{1}<0\right\} \leqq C|w|_{2+k,\left\{x_{1}<0\right\}} .
$$

Combining (5.13), (5.14) and (5.15), let us take $\mu \rightarrow 0$. Then we obtain the estimate (5.2).

Now let us define $w$ in substitute of (5.4) in such a way that for $0 \leqq m \leqq k+1$

$$
D_{x_{1}}^{m} w=\left\{\begin{array}{ccc}
-2 D_{x_{1}}^{m} u & \text { on } \quad x_{1}=0 \text { for even } m \\
0 & \text { on } \quad x_{1}=0 \text { for odd } m .
\end{array}\right.
$$

And we put

$$
v\left(x_{1}, x_{2}, t\right)=-u\left(-x_{1}, x_{2}, t\right)-w\left(x_{1}, x_{2}, t\right) \quad \text { for } \quad x_{1} \leqq 0 .
$$

Then we have the following proposition quite similarly to the proof of Proposition 5.2.

Proposition 5.3. Under the assumptions of Proposition 5.2, if $k \geqq k_{0}$, there is a constant $C$ such that

$$
\begin{aligned}
|u|_{k+2, \Sigma_{+}} \leqq & C\left(|L u|_{k, \Sigma_{+}}+\langle u\rangle_{k+\frac{3}{2}, \Gamma}\right. \\
& \left.+\left\langle u_{x_{2}}+a u_{x_{1}}\right\rangle_{k+\frac{1}{2},\left\{x_{1}>0\right\}}+|u|_{0}\right)
\end{aligned}
$$


for any $u \in C_{0}^{\infty}\left(\Sigma_{+}\right)$.

Further we can prove more easily without using the mixed boundary estimate

Proposition 5.4. Under the same assumptions, if $k \geqq 0$, it holds that for any $u \in C_{0}^{\infty}\left(\bar{\Sigma}_{+}\right)$

$$
|u|_{k+2, \Sigma_{+}} \leqq C\left(|L u|_{k, \Sigma_{+}}+\langle u\rangle_{k+\frac{3}{2}, \Gamma}+\langle u\rangle_{k+\frac{3}{2},\left\{x_{1}>0\right\}}+|u|_{0}\right) .
$$

And we can take $k_{0}=0$ in the assumptions of Proposition 5.2 and 5.3, if $a=0$ in (5.2) and (5.16) respectively.

It is easily seen that the estimates (5.2), (5.16) and (5.17) hold also for the operator

$$
\left(1+\delta_{1}\right) \frac{\partial^{2}}{\partial x_{1}^{2}}+\delta_{2} \frac{\partial^{2}}{\partial x_{1} \partial x_{2}}+\left(1+\delta_{3}\right) \frac{\partial^{2}}{\partial x_{2}^{2}}+e^{i \theta} \frac{\partial^{2}}{\partial t^{2}}
$$

if $\delta_{1}, \delta_{2}$ and $\delta_{3}$ are sufficiently small positive numbers.

Now the closed graph theorem was used only to prove Proposition 3.1 ([19]). And Proposition 3.1 is needless to the proof for the case $a=0$ in Propositions 5.2, 5.3 and 5.4. Therefore the admissible value of $\delta_{1}, \delta_{2}$ and $\delta_{3}$ for the operator (5.18) can be calculated exactly. Thus performing a coordinate transformation, we see

Proposition 5.5. There is a positive constant $\Theta_{0}$ such that the statement of Propositions 5.2, 5.3 and 5.4 holds also for the angular domain with the angle $\frac{\pi}{2}+\alpha\left(|\alpha|<\Theta_{0}\right)$ in the place of $\Sigma_{+}$. In particular, we can compute the constant $\Theta_{0}$ explicitly for the case of $a=0$ in Propositions 5.2, 5.3 and the case of Proposition 5.4.

6. Let us return to the section 1 . We assume that $A, B_{j}, \mathscr{D}$ and $\theta$ satisfy the assumption of Theorem 1 . Introducing a new real variable $t$, we set

$$
\tilde{A}=A+e^{i \theta} \frac{\partial^{2}}{\partial t^{2}}
$$


Let us denote by $\mathscr{D} \times\{t\}$ the 3-dimensional domain $\left\{\left(x_{1}, x_{2}, t\right) \mid\left(x_{1}, x_{2}\right) \in \mathscr{D}\right.$, $-\infty<t<\infty\}$.

The passage from Proposition 5.5 with constant coefficients and with angular domains to the following proposition is performed in a familiar method based on a partition of unity (cf., e.g., $[1],[3],[20]$ ). We denote by $C_{0}^{\infty}(\overline{\mathscr{D}} \times\{t\})$ the class of $u \in C^{\infty}(\overline{\mathscr{D}} \times\{t\})$ vanishing for $|t|$ larger than some fixed number. Then we have

Proposition 6.1. Under the assumptions of Theorem 1 there is a constant $k_{0}$ such that for any $u \in C_{0}^{\infty}(\overline{\mathscr{D}} \times\{t\})^{8)}$

$$
\begin{aligned}
& \|u\|_{k+2, \mathscr{D} \times\{t\}} \leqq C\left(\|\tilde{A} u\|_{k, \mathscr{D} \times\{t\}}\right. \\
& \left.+\sum_{j}\left\langle B_{j} u\right\rangle_{\frac{3}{2}-m_{j+k}, \Gamma_{j} \times\{t\}}+\|u\|_{0, \mathscr{D} \times\{t\}}\right),
\end{aligned}
$$

if $k \geqq k_{0}$.

Now we shall deduce Theorem 1 from Proposition 6.1. The method is essentially analogous to that of Agmon [2]. He proved for the case that the boundary values vanish. And his proof is effective for the $L^{p}$ norm. First we prepare the following lemma.

Lemma 6.1. Let $\zeta(t)$ be a fixed function in $C_{0}^{\infty}\left(R^{1}\right)$. We set $v_{\mu}(x, t)=\zeta(t) e^{i \mu t} v(x)$ for any $v$ in $C_{0}^{\infty}\left(R^{1}\right)$ and for any real $\mu \geqq 1$. Then for given real $s \geqq 0$, there is a constant $C$ such that

$$
\left|v_{\mu}\right|_{s, R^{2}} \leqq C\left(|v|_{s}+\mu^{s}|v|_{0}\right) .
$$

Proof. We see

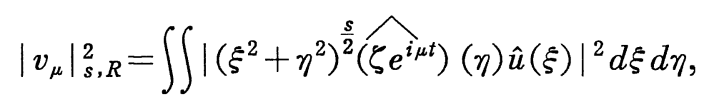

where $\wedge$ represents the Fourier transform.

Obviously,

$$
\left.\widehat{\left(\zeta e^{i \mu t}\right.}\right)=\hat{\zeta}(\eta-\mu)
$$

And we have

8) Put $\overline{\mathscr{D}} \times\{t\}=\left\{\left(x_{1}, x_{2}, t\right) \mid\left(x_{1}, x_{2}\right) \in \overline{\mathscr{D}},-\infty<t<\infty\right\}$. 


$$
\begin{aligned}
\left(\xi^{2}+\eta^{2}\right)^{\frac{s}{2}} & \leqq C\left(|\eta-\mu|^{2}+\xi^{2}+\mu^{2}\right)^{\frac{s}{2}} \\
& \leqq C\left(1+|\eta-\mu|^{2}\right)^{2}\left(1+\mu^{2}+\xi^{2}\right)^{\frac{s}{2}} \\
& \leqq C\left(1+|\eta-\mu|^{2}\right)^{2}\left(|\xi|^{s}+\left(1+\mu^{2}\right)^{\frac{s}{2}}\right) .
\end{aligned}
$$

Hence,

$$
|v|_{s, R^{2}}^{2} \leqq C \int\left(1+\eta^{2}\right)^{s}|\xi(\eta)|^{2} d \eta \int\left(|\xi|^{s}+\left(1+\mu^{2}\right)^{\frac{s}{2}}\right)^{2}|\hat{v}(\xi)|^{2} d \xi .
$$

Thus we obtained the proof.

Lemma 6.2. Under the assumptions of Lemma 6.1, we have

$$
\left|v_{\mu}\right|_{s, R_{+}^{2}} \leqq C\left(|v|_{s, R_{+}^{1}}+\mu^{s}|v|_{0, R_{+}^{1}}\right)
$$

where ||$_{s, R_{+}^{2}}$ and ||$_{s, R_{+}^{1}}$ are the quotient semi-norms in $\{x>0\}$, respectively.

Proof. We consider the function

$$
\tilde{v}(x)= \begin{cases}v(x) & \text { in } x \geqq 0 \\ \sum_{p=1}^{[s]+1} \lambda_{p} v(-p x) & \text { in } \quad x<0 .\end{cases}
$$

If we choose $\lambda_{p}$ adequately, then $\tilde{v}(x)$ belongs to $C_{0}^{[s]+1}\left(R^{1}\right)$. Since the quantity $|u|_{s, R_{+}^{1}}$ can be written by the double integral, we see

$$
|\tilde{v}|_{s} \leqq C|v|_{s, R_{+}^{1}}
$$

And obviously,

$$
|\tilde{v}|_{0} \leqq C|v|_{0, R_{+}^{1}}
$$

Put $\widetilde{v}_{\mu}(x, t)=\zeta(t) e^{i \mu t} \tilde{v}(x)$. Then we easily see

$$
|v|_{s, R_{+}^{2}} \leqq\left|\tilde{v}_{\mu}\right|_{s, R^{2}}
$$

Combining (6.1), (6.2), (6.3) and Lemma 6.1 we have the desired inequality.

Proof of Theorem 1.

We take a function $\zeta(t) \in C_{0}^{\infty}\left(R^{1}\right)$ in such a way that 


$$
\zeta(t)=\left\{\begin{array}{lll}
0 & \text { if } & |t|>1 \\
1 & \text { if } & |t|<1 / 2 .
\end{array}\right.
$$

Let us set $v_{\mu}\left(x_{1}, x_{2}, t\right)=\zeta(t) e^{i \mu t} u(x)$ for any $u \in C^{\infty}(\overline{\mathscr{D}})$ and any real $\mu(\geqq 1)$. Then we see by Proposition 6.1

$$
\begin{aligned}
\left\|v_{\mu}\right\|_{k+2, Q_{1}} \leqq & C\left(\left\|\tilde{A} v_{\mu}\right\|_{k, Q_{1}}+\left\|v_{\mu}\right\|_{0, Q_{1}}\right. \\
& \left.+\sum_{j}\left\langle B_{j} v_{\mu}\right\rangle \frac{3}{2}-m_{j}+k, \partial_{Q_{r, j}}\right)
\end{aligned}
$$

where $Q_{r}=\mathscr{D} \times\{|t| \leqq r\}$ and $\partial Q_{r, j}=\Gamma_{j} \times\{|t| \leqq r\}$.

Clearly,

$$
\begin{aligned}
\tilde{A} v_{\mu}= & \zeta(t) e^{i \mu t}\left(A-\mu^{2} e^{i \theta}\right) u \\
& +e^{i \theta}\left(\zeta^{\prime \prime}(t) e^{i \mu t}+2 \zeta^{\prime}(t) i \mu e^{i \mu t}\right) u .
\end{aligned}
$$

Hence if we put $D^{\alpha}=D_{x_{1}}^{\beta_{1}} D_{x_{2}}^{\beta_{2}} D_{t}^{\gamma}=D_{x}^{\beta} D_{t}^{\gamma}$,

$$
\begin{aligned}
D^{\alpha} \tilde{A} v_{\mu}= & D_{t}^{\gamma}\left(\zeta e^{i \mu t}\right) D_{x}^{\beta}\left(\left(A-\mu^{2} e^{i \theta}\right) u\right) \\
& +e^{i \theta} D_{t}^{\gamma}\left(\zeta^{\prime \prime} e^{i \mu t}+2 \zeta^{\prime} i \mu e^{i \mu t}\right) D_{x}^{\beta} u .
\end{aligned}
$$

Accordingly,

$$
\begin{aligned}
\left\|\tilde{A} v_{\mu}\right\|_{k, Q_{1}} \leqq & C\left(\sum_{\gamma=0}^{k} \mu^{2 \gamma}\left\|\left(A-\mu^{2} e^{i \theta}\right) u\right\|_{k-\gamma}^{2}\right. \\
& \left.+\sum_{\gamma=0}^{k} \mu^{2 \gamma+2}\|u\|_{k-\gamma}^{2}\right) .
\end{aligned}
$$

It is easily seen that (see e.g. $[5],[10]$ )

$$
\begin{aligned}
\mu^{2 \gamma}\left\|\left(A-\mu^{2} e^{i \theta}\right) u\right\|_{k-\gamma}^{2} \leqq & C\left(\left\|\left(A-\mu^{2} e^{i \theta}\right) u\right\|_{k}^{2}\right. \\
& \left.+\mu^{2 k}\left\|\left(A-\mu^{2} e^{i \theta}\right) u\right\|_{0}^{2}\right) .
\end{aligned}
$$

On the other hand, we see from Lemmas 6.1, 6.2 and the definition of the boundary norm

$$
\begin{aligned}
\left\langle B_{j} v_{\mu}\right\rangle_{\frac{3}{2}-m_{j}+k, \partial Q_{1, j} \leqq} & C\left(\left\langle B_{j} u\right\rangle_{\frac{3}{2}-m_{\jmath}+k, \Gamma_{j}}\right. \\
& \left.+\mu^{\frac{3}{2}-m_{j+k}}\left\langle B_{j} u\right\rangle_{0, \Gamma_{j}}\right) .
\end{aligned}
$$

In addition, 


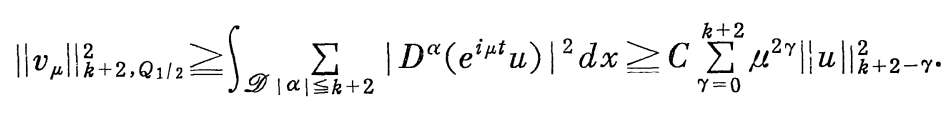

Combining (6.4), (6.5), (6.6), (6.7) and (6.8) we have completed the proof.

Finally since the constant $k_{0}$ can be taken to be 0 for Proposition 5.4, the statement of Theorem 2 has been shown.

\section{References}

[1] Agmon, S., A. Douglis and L. Nirenberg, Estimates near the boundary for solutions of elliptic partial differential equations satisfying general boundary conditions I, Comm. Pure Appl. Math. 12 (1959), 623-727.

[2] Agmon, S., On the eigenfunctions and on the eigenvalues of general elliptic boundary value problems, Comm. Pure Appl. Math. 15 (1962), 119-147.

[3] Agmon, S., General elliptic boundary value problems, Nostrand, 1965.

[4] Agranovich, N. and M. I. Vishik, Elliptic problems with a parameter and parabolic problems of general type, Uspehi Mat. Nauk 19 (1964), Russian Math. Surveys 19 (1964), 53-157.

[5] Browder, F.E., On the spectral theory of elliptic differential operators I, Math. Ann. 142 (1961), 22-130.

[6] Gårding, L., Dirichlet's problem for linear elliptic partial differential equations, Math. Scand. 1 (1953), 55-72.

[7] Gohberg, I. C. and M. G. Krein, Systems of singular integral equation on the half line with kernels depending on the arguments, Uspehi Mat. Nauk 13 (1958), 3-72.

[8] Gohberg, I. C., Factorization problems in normed rings, Uspehi Mat. Nauk 19 (1964), 71-124.

[9] Hanna, M. S. and K. T. Smith, Some remarks on the Dirichlet problem in piecewise smooth domains, Comm. Pure Appl. Math. 20 (1967), 575-593.

[10] Higuchi, Y., A priori estimates and existence theorem on elliptic boundary value problems for unbounded domains, Osaka J. Math. 5 (1968), 103-135.

[11] Hörmander, L., Linear partial differential operators, Springer, 1963.

[12] Kadlec, J., The regularity of the solution of the Poisson problem in a domain whose boundary is similar to that of a convex domain, Czechoslovak Math. J. 89 (1964), 386-393.

[13] Ladyzhenskaya, O. A., A simple proof of the solvability of the fundamental boundary value problems and the eigenvalue problem for linear elliptic equations, Vestnik Leningrad Univ. Mat. Mekh. Astronom. 11 (1955), 23-29.

[14] Ladyzhenskaya, O. A., On integral estimates, convergence of approximation methods and solutions in functionals for linear elliptic operators, Vestnik Leningrad Univ. Mat. Mekh. Astronom. 14 (1958), 60-69.

[15] Lions, J. L. and E. Magenes, Problèmes aux limites non homogènes (II), Ann. Inst. Fourier Grenoble 11 (1961), 137-178.

[16] Lions, J. L. and E. Magenes, Problèmes aux limites non homogènes (IV), Annali Scoula Norm. Sup. Pisa 15 (1961), 311-326.

[17] Peetre, J., Mixed problems for higher order elliptic equations in two variables (I), Annali Scoula Norm. Sup. Pisa 15 (1961), 337-353.

[18] Shamir, E., Mixed boundary value problems for elliptic equations in the plane, 
Annali Scuola Norm. Sup. Pisa 17 (1963), 117-138.

[19] Shamir, E., Elliptic systems of singular integral operators I, Trans. Amer. Math Soc. 39 (1968), 107-124.

[20] Schechter, M., Integral inequalities for partial differential operators and functions satisfying general boundary conditions, Comm. Pure Appl. Math. 12 (1959), 37-66.

[21] Schechter, M., General boundary value problems for elliptic differential operators, Comm. Pure Appl. Math. 12 (1959), 457-486.

[22] Schechter, M., Mixed boundary problems for general elliptic equations, Comm. Pure Appl. Math. 13 (1960), 407-425.

[23] Vishik, M. I. and G. I. Eskin, General boundary value problems with discontinuous conditions at the boundary, Dokl. Akad. Nauk SSSR 158 (1964), 15-19.

[24] Vishik M. I. and G. I. Eskin, Elliptic equations in convolution in a bounded domain and their applications, Uspehi Mat. Nauk 22 (1967), Russian Math. Surveys 22 (1967), 15-124.

[25] Zygmund, A., Trigonometrical series I, Cambridge, 1959. 